\title{
Una Mirada Hacia el Interior DEl Subsistema de UNIVERSIDADES TeCNOLÓgICAS (SuT)*
}

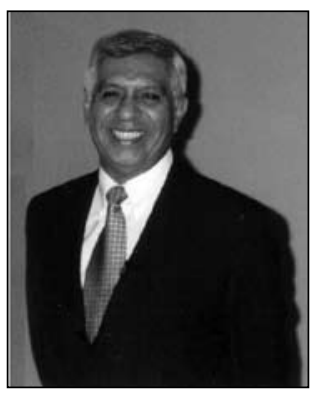

Dr. Arturo Nava Jaimes

Director General del SUT.

Coordinación General de Universidades

Tecnológicas del la secretaria de Educación

Pública, México

E-mail:anava@cgut.sep.gob.mx

\begin{abstract}
Na entrevista concedida a Rafael Hernández e Guadalupe
Bueno, o dr. Nava discorre sobre o Subsistema de Universidades Tecnológicas (SUT) do México, que é o sistema educativo de nivel superior que mais se desenvolveu nos últimos 16 anos no país. Implantado em 1991, hoje conta com 61 instituicooes, presentes em 26 dos 32 estados do país. O SUT oferece atualmente formação em 30 tipos de carreiras, em diferentes dominios como as engenharias, a administração e os serviços. Todos os cursos têm duração de dois anos e se caracterizam por ter uma área de formação básica, uma técnica específica, própria da carreira, e outra flexivel, de acordo as características da região onde se encontra a Universidade Tecnológica (UT). A singularidade da UT consiste em oferecer grades com carga horária integral, que dão resposta às necessidades do setor produtivo. Assim, os atributos da educação universitário-tecnológica orientam-se à polivalência, à intensidade da formação, à continuidade dos estudos, à flexibilidade dos planos e grades, $e$ à pertinência às necessidades reais de produção. É importante destacar que esse modelo educativo deu oportunidade de acesso à educação superior universitária a grupos de zonas urbanas e rurais tradicionalmente excluidos. Dessa perspectiva, a educação pública tecnológica se caracteriza pela atenção educativa a alunos com relativa desvantagem social e econômica, buscando promover a eqüidade na oferta de formação profissional. $O \mathrm{dr}$. Nava, é o diretor-geral do SUT e responsável pelo desenvolvimento do subsistema desde sua implantação.
\end{abstract}

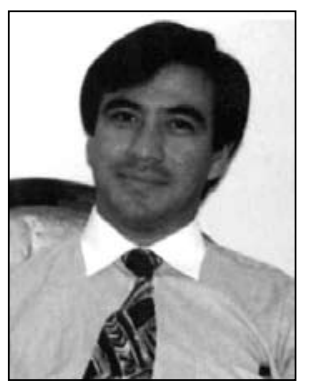

Rafael Hernández Director del Área Ciencias de la Vida y la Conducta, CENEVAL, México E-mail: rafael.hernandez@ceneval.edu.mx

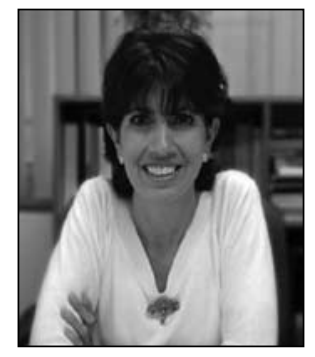

Guadalupe Vadillo Bueno. Secretaria del Consejo Técnico de Bachillerato. Universidad Nacional Autónoma de México E-mail:gvadillo@servidor.unam.mx
Entrevistador (E.): Dr. Nava, antes de empezar esta entrevista, le agradezco su amabilidad por aceptar y el tiempo que nos otorga para realizarla. La primera pregunta, a modo de introducción es: ¿Qué idea originó las UT?

Dr. Nava (Dr. N.): La idea de crear las Universidades Tecnológicas proviene en primera instancia de la necesidad de encontrar alternativas de educación superior que cubrieran la creciente demanda de egresados de bachillerato. Por un lado, las instituciones de educación superior, se vieron paulatinamente rebasadas en su capacidad de absorción de estudiantes provenientes del nivel medio superior. Por el otro, los modelos tradicionales de * Elpresente documento forma parte del trabajo de investigación que el primer autor realiza
para obtener el grado de doctor en Educación por la Universidad La Salle. formación propedéutica de nivel medio superior, aunada con el modelo de formación de licenciatura, de cuatro a cinco años y con una fuerte carga teórica, no coincidían con las necesidades sociales de inserción laboral de corto plazo y una formación relacionada con las necesidades de los empleadores. Es entonces que se realiza un esfuerzo por conocer cómo se desarrollan los sistemas educativos de otros países, a fin de identificar las prácticas estructurales que permitirían eventualmente incorporar nuevos esquemas de formación de nivel superior.

E: Sabemos que en otras parte del mundo existen sistemas educativos similares, tal es el caso de los Estados Unidos de América, en donde existen los community colleges; en Francia, los Institutos Tecnológicos; o en España, las Universidades Tecnológicas. ¿Cuál es la diferencia más 
importante con las instituciones del mismo nivel en otros países?

Dr. N.: En esencia, la formación que se ofrece de este tipo en diferentes países es similar, considerando que corresponden al nivel 5B de la Clasificación Internacional Normalizada de la Educación (Cine), de la Unesco, la cual corresponde a formación profesional en el nivel terciario (educación superior), que se caracteriza por su corta duración, orientada a la práctica y dirigida a la formación de habilidades para la inserción laboral. La diferencia radica principalmente en la estructura interna de operación y su articulación dentro del sistema educativo en el país respectivo. Por ejemplo, la carga horaria en las Universidades Tecnológicas es en promedio de 35 horas semanales, mientras que en otros países dicha carga no excede las 20 horas. Por otro lado, las Universidades Tecnológicas forman un subsistema de educación superior en México y la movilidad estudiantil se realiza a través de los convenios establecidos con otros subsistemas e instituciones en lo particular, mientras que, en Francia o Estados Unidos, los niveles 5B y 5A (correspondiente a licenciatura) se encuentran integrados en la misma institución.

\section{E: ¿Cómo fue el proceso de planeación de las Universidades Tecnológicas?}

Dr. N.: Las primeras acciones consistieron en un proceso de diagnóstico de la educación en México, en el cual se identificó una demanda creciente por el acceso a estudios de nivel superior, así como una necesidad de incorporación al mercado laboral, lo cual produce abandono escolar. Por otro lado, se realizó un análisis de las opciones de educación superior que se ofrecen en otros países, donde se identifica esta modalidad, que en México no había sido explorada.

\section{Las primeras acciones} consistieron en un proceso de diagnóstico de la educación en México, en el cual se identificó una demanda creciente por el acceso a estudios de nivel superior, así como una necesidad de incorporación al mercado laboral, lo cual produce abandono escolar.
El análisis de las modalidades de educación superior de dos a tres años después del bachillerato se hizo con detenimiento y se consideró que el que mejor se podía adaptar al Sistema Educativo Mexicano era el modelo francés. Con ello se procedió al diseño del modelo educativo, que inició operaciones en 1991 con tres Universidades Tecnológicas: Aguascalientes, Nezahualcóyotl y Tula Tepeji. Aún antes de decidir su expansión, se tomó la decisión de observar el desarrollo alcanzado en los primeros planteles. La decisión de expandir el subsistema se tomaría hasta que las tres primeras demostraran su viabilidad, para lo cual habría que esperar a que egresara la primera generación y observar sus resultados en la inserción laboral.

E: ¿Qué datos sustentaban la necesidad de que se constituyeran? y ¿cuántas unidades se planearon inicialmente?

Dr. N.: Antes de revisar cifras respecto a la necesidad de las Universidades Tecnológicas, sería importante analizar las teorías acerca de la relación entre la educación y el desarrollo de las sociedades. Por ejemplo, la teoría del capital humano sustenta que el desarrollo económico de las sociedades está fuertemente ligado a la preparación académica de los individuos que las conforman. Aunque esta corriente en la actualidad no es suficiente para explicar muchos fenómenos, como el desempleo creciente, es un punto de partida para la inversión en educación. A nivel individual, baste el razonamiento natural que a mayor preparación, mejores oportunidades de elevar la calidad de vida. Visto así, los esfuerzos realizados por prácticamente cualquier país en materia de educación traen consigo un principio de utilidad de la educación en la calidad de vida de las personas y de las sociedades. México no ha sido ajeno a estos principios y, a partir del diagnóstico realizado, se identificó la necesidad de diversificar las alternativas de educación superior para atender la demanda creciente.

Algunos datos se pueden encontrar en estudios realizados por Instituto Nacional de Estadística, Geografía e Informática (INEGI), respecto al crecimiento de los niveles de escolaridad en la sociedad mexicana y la relación entre la escolaridad y el empleo. Asimismo la Asociación Nacional de Universidades e Instituciones de Educación Superior (Anuies) cuenta con anuarios estadísticos que revelan cifras como el abandono escolar en el nivel de licenciatura.

E.: ¿Cuáles fueron las principales dificultades durante la etapa de planeación? Considerando que se realizan estu- 
dios técnicos y socioeconómicos para abrir las UT, ¿cuál es la principal diferencia entre ellas? ¿Cuáles se abrieron por insistencia de las autoridades estatales, municipales o de la misma comunidad, aunque no se cumplieran los requisitos para abrirla, por ejemplo, el caso de la del Norte de Aguascalientes?

Dr. N.: Para hablar de las dificultades de un proyecto a nivel nacional, tendríamos que considerar primeramente que las Universidades Tecnológicas son organismos públicos descentralizados de los gobiernos de los estados. Es decir, cualquier universidad surge como una iniciativa de los gobiernos estatales y se rige por la normatividad estatal en materia de educación. Sin embargo la participación federal en cuanto al recurso es del $50 \%$ y, por otro lado, su función es la de vigilar que se aplique el modelo educativo, tal y como fue concebido para este subsistema de educación superior.

Las dificultades en el proceso de planeación radicaron al inicio en establecer una metodología unificada que permitiera identificar las necesidades del entorno bajo las cuales se determinaría la oferta educativa de la universidad en operación. Por otro lado, las primeras universidades dejarían ver las limitantes de instrumentar el modelo educativo, tales como la contratación del personal docente que cubriera el perfil para impartir clases. En tanto se trataba de un modelo novedoso, no había personal docente que contara con estas características, por lo que el proceso de formación fue uno de los aspectos centrales a desarrollar. La primera generación de universidades contribuyó fuertemente al proceso de instrumentación sistemática de diversos procesos académicos y organizacionales, que aunque estaban debidamente planeados, requerían formar al personal dentro de la filosofía del modelo educativo.

La diferencia entre las universidades que iniciaron el proyecto radica principalmente en su entorno. Los estudios de factibilidad determinaron una oferta específica para cada caso y su entorno promovió las características particulares de operación del modelo educativo. Actualmente se pueden distinguir en lo general dos tipos de entorno: uno industrializado, en el que se identifica con facilidad el tipo de profesional requerido, su perfil, las necesidades prevalecientes entre las empresas y el tipo de actividad económica predominante. En otro polo se encuentran las universidades cuyo entorno es más limitado y cuya función es favorecer el desarrollo regional.

Respecto a la insistencia de los gobiernos estatales, todas las Universidades Tecnológicas cuentan con el debido sustento para su creación. La coordinación general de Universidades Tecnológicas dictamina, a partir de los estudios de factibilidad, la viabilidad de las universidades a crear y todas sin excepción cuentan con estos estudios. Inclusive se cuenta con evidencia de los proyectos que han sido rechazados por carecer de sustento. La UT del Norte de Aguascalientes surgió primeramente como una unidad académica de la Universidad Tecnológica de Aguascalientes y su creación vino precedida de su operación como unidad académica. Una vez que se logró sustentar su viabilidad para funcionar como universidad, se estableció con los mismos requisitos que a cualquier otra del subsistema.

E.: ¿Por qué ha crecido de manera tan importante el subsistema? ¿A qué atribuye el apoyo para el crecimiento del subsistema?

Dr. N.: Las Universidades Tecnológicas, desde su creación, se han sometido a diversos procesos de auto evaluación y evaluación externa, que han demostrado la viabilidad del proyecto. Los indicadores de resultados y la revisión de sus procesos siempre han estado al alcance de autoridades, expertos evaluadores extranjeros $\mathrm{y}$, en los últimos años, se ha abierto al escrutinio de diversos investigadores, que han realizado aportaciones importantes para la mejora continua del modelo. Por otro lado, ha desarrollado sistemas de auto evaluación institucional y del subsistema, a partir de estadísticas, indicadores y estudios que permiten realizar ajustes a su política educativa. Las Universidades Tecnológicas se han mantenido en constante evolución, por lo que se insertan con oportunidad a los proyectos prioritarios de las autoridades educativas y en ocasiones han sido motor para generar procesos de desarrollo y actualización en otros subsistemas de educación superior.

El crecimiento de las Universidades Tecnológicas, aunque en ciertas etapas ha sido acelerado, siempre ha mantenido un sentido de orden. No se crean universidades sin antes ubicarse dentro de un proyecto de desarrollo educativo.

E.: ¿Cómo compara su crecimiento con el de otras instituciones educativas de carácter público?

Dr. N.: Las Universidades Tecnológicas se han orientado a la atención de población que difícilmente tendría otras oportunidades de acceso a la educación superior. En ese sentido, la cobertura con equidad ha sido un aspecto fundamental del crecimiento de las Universidades Tecnológicas. Otros subsistemas ya tienen una

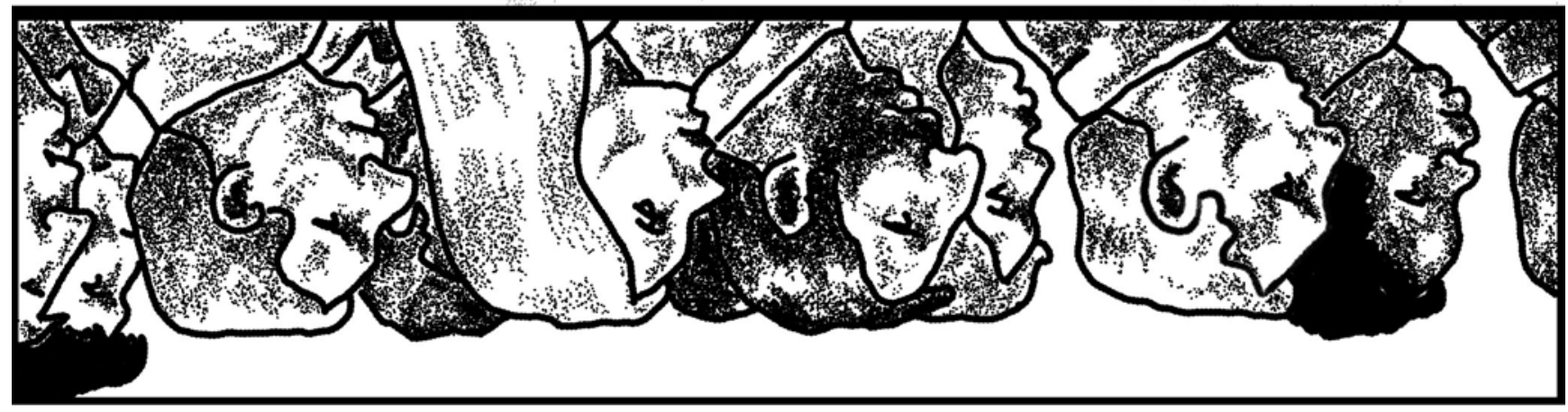


matrícula relativamente estable y aunque su crecimiento en términos absolutos podría ser superior debido a la creciente demanda de educación superior, las Universidades Tecnológicas se han logrado ubicar como una opción considerablemente importante. Podemos citar que uno de cada cuatro estudiantes en las Universidades Tecnológicas no consideraron la posibilidad de estudiar en el nivel superior, de no existir esta alternativa de estudios. Existen casos como la Universidad Tecnológica de Querétaro, que se ha constituido como la tercera institución con mayor matrícula en el estado, sólo superada por la universidad pública estatal y un instituto tecnológico que se ubica en la capital.

E.: ¿En comparación con las otras IES de carácter público, ¿usted considera que las UT, por sus características, constituyen un subsistema más homogéneo?

Dr. N.: Es responsabilidad de cualquier subsistema vigilar que sus elementos componentes se desarrollen con base en ciertas pautas básicas de operación, tanto en los aspectos educativos, como administrativos. En el caso de las Universidades Tecnológicas, existen aspectos que se vigilan por atribuciones conferidas a la coordinación general de Universidades Tecnológicas, pero más importante aún, muchos de los proyectos que se realizan en el subsistema se deciden por consenso de los rectores. El desarrollo del subsistema siempre ha contado con la toma de decisiones en conjunto y la asunción de responsabilidades compartidas, generadas por consenso, no por votación, entre los rectores de todas las Universidades. Para ello se realizan periódicamente reuniones de rectores, en las cuales se toman acuerdos y, cuando es necesario, se forman comisiones para desarrollar los proyectos. También se cuenta con comisiones académicas y de vinculación, que revisan permanentemente el diseño curricular del modelo, donde participan los directores de carrera y secretarios académicos y de vinculación de las UT.

E.: Doctor, en cuanto a la planta docente de las UT, ¿cómo se constituyó? ¿Cuáles fueron sus principales dificultades para aplicar el modelo educativo? ¿Se ha realizado un seguimiento longitudinal de su desempeño?

Dr. N.: El modelo educativo se planeó para que los profesores pudieran ofrecer una atención personalizada a los alumnos. Es por ello que los grupos son de 25 alumnos como máximo. Por cada 25 alumnos, se asigna un profesor de tiempo completo y se designan 20 horas cubiertas por profesores de asignatura. La característica fundamental de los profesores es que cuenten con experiencia en el campo profesional y, en el caso de los profesores de asignatura, deberán laborar en el momento de impartir clases en el sector productivo relacionado con la asignatura que imparten. El perfil mínimo aceptable es el de licenciatura y el mínimo deseable es el de maestría o especialidad tecnológica. Los profesores de tiempo completo realizan funciones complementarias a la docencia, como gestión académica y de vinculación, tutorías y asesorías, y desarrollo de líneas de aplicación pertinente del conocimiento. Actualmente se conforman cuerpos académicos, que permitirán una integración del personal docente por áreas de desarrollo prioritarias para las instituciones.
La evolución del desempeño de los docentes corresponde en un sentido a las propias universidades, que tienen la responsabilidad de evaluar continuamente este aspecto y ofrecer a sus profesores, oportunidades de actualización y formación docente, a partir de los resultados de dicha evaluación.

La evolución del desempeño de los docentes corresponde en un sentido a las propias universidades, que tienen la responsabilidad de evaluar continuamente este aspecto y ofrecer a sus profesores, oportunidades de actualización y formación docente, a partir de los resultados de dicha evaluación. En otro sentido, la coordinación general cuenta con ejercicios de auto evaluación institucional y con indicadores de desempeño, plasmados en el modelo de evaluación de la calidad que aplican sistemáticamente las UT. Además, se realizan estudios que miden periódicamente el desempeño global del profesorado de las UT, por medio de las opiniones de los alumnos.

E.: Doctor, para finalizar esta entrevista, nos podría decir cuál ha sido el mayor logro de este subsistema? ¿Cuál es su mayor contribución social? ¿Cuál es su mayor fortaleza?

Dr. N.: Sería poco prudente establecer en una escala cuál ha sido el mayor logro de las UT. Sin embargo, en la más reciente evaluación, realizada al subsistema en el 2006, por expertos franceses, resaltan las siguientes fortalezas:

- calidad del egresado;

- fuerte apoyo a la movilidad social y la equidad (que podría ser una de sus contribuciones sociales más importantes);

- modelo educativo con enfoque práctico;

- cultura de la calidad y la evaluación;

- satisfacción de sus actores;

- mejoramiento del cuerpo docente;

- desarrollo significativo de la vinculación; y

- mejoramiento de la gobernabilidad del subsistema.

Estas fortalezas reflejan el esfuerzo por mantener la integración del subsistema en el desarrollo y operación de las Universidades Tecnológicas. 